\title{
The Medial Temporal Lobe Distinguishes Old from New Independently of Consciousness
}

\author{
Sander M. Daselaar, ${ }^{1,2}$ Mathias S. Fleck, ${ }^{2,3}$ Steven E. Prince, ${ }^{2,3}$ and Roberto Cabeza ${ }^{2,3}$ \\ ${ }^{1}$ University of Amsterdam, Faculty of Science, Swammerdam Institute for Life Sciences, 1098 SM Amsterdam, The Netherlands, and ${ }^{2}$ Duke University, \\ Center for Cognitive Neuroscience and ${ }^{3}$ Department of Psychological and Brain Sciences, Durham, North Carolina 27708
}

\begin{abstract}
Although it is widely accepted that the medial temporal lobes (MTLs) are critical for becoming aware that something happened in the past, there is virtually no evidence whether MTL sensitivity to event oldness also depends on conscious awareness. Using event-related functional magnetic resonance imaging, we show that activity in posterior MTL tracks whether an item is actually old (true oldness), regardless of participants' awareness of oldness (perceived oldness). Confirming its sensitivity to the objective nature of the stimulus, activity in this region was strongly correlated with individual memory performance $(r=0.74)$. At the same time, we found that memory errors (misses) were associated with activity in an anterior MTL region, which signaled whether an item was consciously experienced as new (perceived novelty). Logistic regression analyses based on individual trial activity indicated that the two MTL regions showed opposing relationships with behavior, and that memory performance was determined by their joint activity. Furthermore, functional connectivity analyses showed that perceived novelty activity in the posterior MTL inhibited true oldness activity in the anterior MTL. These findings indicate that participants' behavior reflected the combined effects of multiple MTL regions. More generally, our results show that parts of MTL can distinguish old from new independently of consciousness.
\end{abstract}

Key words: behavior; cognition; memory; recognition; human; hippocampus; parahippocampal

\section{Introduction}

One of the most fundamental memory functions is the ability to determine whether or not a present event happened in the past; that is, the ability to distinguish old from new. It is generally agreed that conscious awareness of the oldness of an event depends on the integrity of the medial temporal lobes (MTLs) (Moscovitch, 1995; Eichenbaum, 2004; Squire et al., 2004). In support of this idea, functional neuroimaging studies have directly related MTL activity to conscious memory processes (Schacter et al., 1996; Eldridge et al., 2000). However, there is virtually no evidence about whether MTL sensitivity to the oldness of stimuli necessarily leads to conscious awareness of oldness. In other words, can MTL activity distinguish old from new information in situations in which we are not consciously aware of the difference? To investigate this question, we scanned young participants with event-related functional magnetic resonance imaging (fMRI) while they were classifying items as old or new.

Participants studied a series of words and, during scanning, they viewed a mixed list of studied (old) and nonstudied (new) words, and classified them as old or new. As illustrated by Figure $1 A$, there are four possible outcomes in this task: a studied item may be correctly classified as old (hit) or incorrectly classified as

\footnotetext{
Received Sept. 28, 2005; revised March 20, 2006; accepted April 18, 2006.

This work was supported by a National Institutes of Health Grant AG19731 to RC. We thank Amber Baptiste Tarter for assistance in subject recruitment and Rakesh Arya for analysis support.

Correspondence should be addressed to Sander M. Daselaar, University of Amsterdam, Faculty of Science, Swammerdam Institute for Life Sciences, Kruislaan 320, 1098 SM Amsterdam, The Netherlands. E-mail: s.m.daselaar@uva.nl.

DOI:10.1523/JNEUROSCI.0258-06.2006

Copyright $\odot 2006$ Society for Neuroscience $\quad$ 0270-6474/06/265835-05\$15.00/0
}

new (miss), and a nonstudied item may be correctly classified as new [correct rejection (CR)] or incorrectly classified as old [false alarm (FA)]. The figure also shows two basic components of recognition memory: whether or not the item or event is actually old or new (true oldness: old vs new stimuli), and whether or not we consciously experience it as old or new (perceived oldness: old vs new responses). These two aspects of memory, objective and subjective, correspond to the two factors crossed in the matrix in Figure $1 A$. To distinguish between these two factors, it is critical to consider all four recognition outcomes (hit, miss, CR, and FA). Yet, the vast majority of functional neuroimaging studies have investigated only hits and CRs (Buckner and Wheeler, 2001), primarily because of limitations in the number of trials required for analyzing fMRI data. In the present study, we were able to collect a sufficient number of trials in all four cells of the matrix, and to distinguish between activity related to true and perceived components of recognition memory.

\section{Materials and Methods \\ Participants}

Fourteen right-handed participants (six female) with an average age of 21.7 (SD, 2.4) were recruited from the Duke University community and paid for participation.

\section{Experimental procedures}

Before scanning, participants studied an intermixed list of 120 real words and 80 pronounceable nonwords while classifying them as real words or nonwords ( $2 \mathrm{~s}$ duration). They knew that their memory for the real words would be tested. Scanning occurred immediately after this study phase.

During scanning, participants performed a recognition memory task and a nonmnemonic perceptual task presented in six blocks (four recog- 
nition and two perceptual, counterbalanced in order across participants). During recognition, participants saw an equal mix of words from the lexical decision task and completely new words ( 60 words per block, $3.4 \mathrm{~s}$ duration), and classified them as old or new. Participants were then prompted to report their confidence (1.7 s) for their answer from 1 (lowest confidence) to 4 (highest confidence), followed by an intertrial interval of $0-5.4 \mathrm{~s}$. In the perceptual task, participants viewed rectangles divided into two sections by a random jagged line. They determined which section had the greater surface area, and then indicated their confidence. Data from the perceptual task are not reported here, and recognition analyses were collapsed across confidence levels to obtain sufficient numbers of trials in the four cells displayed in Figure $1 \mathrm{~A}$.

\section{fMRI procedures}

Functional imaging was performed on a General Electric (Milwaukee, WI) 4T scanner. High resolution T1-weighted structural images [256 $\times 256$ matrix; repetition time (TR), $12 \mathrm{~ms}$; echo time (TE), $5 \mathrm{~ms}$; field of view (FOV), 24 $\mathrm{cm}$; 68 slices; $1.9 \mathrm{~mm}$ thickness] were collected first. Echo-planar functional images were acquired using an inverse spiral sequence $(64 \times$

64 matrix; TR, 1700ms; TE, $31 \mathrm{~ms}$; FOV, 24cm; 34 slices, $3.8 \mathrm{~mm}$ thickness). Scanner noise was reduced with ear plugs and head motion was minimized with foam pads. Stimuli were presented with liquid crystal display goggles (Resonance Technology, Northridge, CA) and responses were registered using a four-key fiber-optic response box (Resonance Technology).

Preprocessing and data analysis were performed using Statistical Parametric Mapping software implemented in Matlab (SPM2; Wellcome Department of Cognitive Neurology, London, UK). Images were slicetiming corrected and motion-corrected, and then spatially normalized to the Montreal Neurological Institute template and smoothed using an 8 $\mathrm{mm}$ kernel. For each subject, trial-related activity was assessed by convolving a vector of trial onsets with a canonical hemodynamic response function. A general linear model (GLM) was specified for each participant to model the effects of interest, generating parameter estimates for hits, misses, CRs, and FAs. Statistical parametric maps were created for each subject by applying linear contrasts to the parameter estimates for these events of interest, resulting in a $t$ statistic for every voxel. A random effects analysis was performed to assess group effects. Focusing on the MTL, the contrasts [(hits and misses) $>$ (CRs and FAs)] (true oldness) and [(hits and FAs) $>$ (misses and CRs)] (perceived oldness) were tested for significance at $p<0.001$, uncorrected, and a 10-voxel cluster size. The mean activity of the MTL regions identified in these analyses was subsequently submitted to a 2 (true oldness) $\times 2$ (perceived oldness) ANOVA.

\section{Follow-up analyses}

Logistic regression analysis. To test the hypothesis that participants' memory performance was codetermined by activity in the anterior and posterior MTL regions identified by the GLM analyses, we conducted a logistic regression analysis based on individual trial activity. As a first step, we created a model in SPM2, in which each individual trial was modeled by a separate covariate, yielding different parameter estimates for each individual trial and for each individual subject (see also, Rissman et al., 2004). The validity of this design was confirmed by the fact that we obtained highly comparable results based on linear contrasts of the single-trial parameter estimates compared with those obtained with a standard model (data not shown). As a second step, mean activity (mean parameter estimates) was extracted for each individual trial from the anterior and posterior MTL regions identified by the true oldness and perceived novelty contrasts. For each individual, the resulting values were then entered into a logistic regression model with activity in the two
MTL regions as independent variables, and a binary variable, which reflected whether participants correctly recognized an old item as old (hit) or incorrectly classified it as new (miss), as the dependent variable. Finally, to assess group effects, the resulting parameter estimates for the anterior and posterior MTL were submitted to a random effects analysis at $p<0.05$.

Dynamic causal modeling analysis. To investigate the functional connectivity between the anterior and posterior MTL, we conducted an analysis using the dynamic causal modeling (DCM) option integrated in SPM2 (Friston et al., 2003). First, we created two volumes of interest (VOIs) using the peaks of the left anterior and posterior MTL, and a 9 $\mathrm{mm}$ radius. Then, for each individual subject, we modeled reciprocal connectivity between the VOIs, and did not modulate connectivity as a function of trial type. Finally, to assess group effects, the resulting connectivity weights $(A)$ were submitted to a random effects analysis at $p<$ 0.05 .

\section{Results}

\section{Behavioral results}

The proportion of hits was 0.71 (SD, 0.14 ), and the proportion of FAs was 0.22 ( $S D, 0.09$ ). The average number of trials for hits, misses, FAs, and CRs, was 86.4 (SD, 16.4), 31.5 (SD, 16.1), 24.2 (SD, 11.2), and 93.3 (SD, 12.4), respectively. The average confidence rating for hits, misses, FAs, and CRs, was 3.25 (SD, 0.30), 2.11 (SD, 0.36), 2.03 (SD, 0.41), and 2.57 (SD, 0.19), respectively. Significant differences in confidence were seen between hits and CRs $(p<0.0001)$, hits and misses $(p<0.0001)$, hits and FAs $(p<0.0001)$, CRs and misses $(p<0.0001)$, and CRs and FAs $(p<0.0001)$.

\section{fMRI results}

The contrasts true oldness [(hit and miss) $>$ (FA and CR $)$ ] and perceived oldness [(hit and FA $)>(\mathrm{CR}$ and miss $)]$ were analyzed. Only the true oldness contrast yielded a significant MTL activation. This activation was found in a posterior parahippocampal/ hippocampal region, bilaterally (Montreal Neurological Institute coordinates: left $x, y$, and $z$ : $-27,-27,-15$; right $x, y$, and $z: 30$, $-23,-15$, respectively). As illustrated by Figure $1 B$, activity in this region was greater for old (hits and misses) than for new 


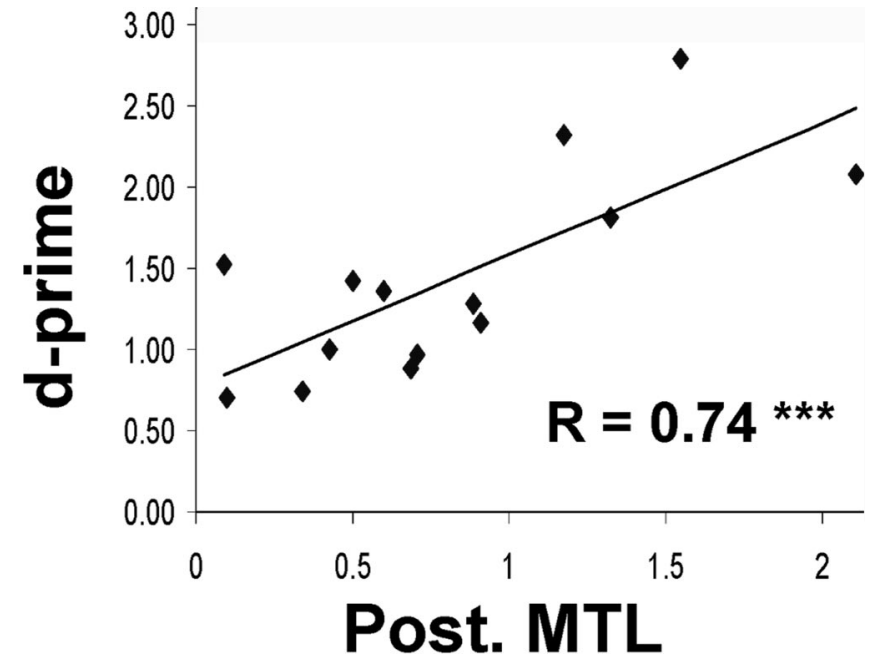

Figure 2. True oldness activity in posterior MTL was strongly correlated with individual memory performance (D-prime).

words (FAs and CRs), regardless of whether they were perceived as old (hits and FAs) or new (CRs and Ms). A 2 (true oldness) $\times$ 2 (perceived oldness) ANOVA yielded a significant effect of true oldness $(p<0.0001)$ and nonsignificant effects of perceived oldness $(p=0.22)$ and true by perceived oldness interaction $(p=$ 0.13 ). Furthermore, as shown in Figure $1 B$, activity in this region was as high for hits as for misses. Confirming this observation, a direct comparison between hits and misses with a paired $t$ test revealed no significant differences in mean cluster activity (left posterior MTL, $p=0.645$; right posterior MTL, $p=0.691$ ). Thus, the results of this study answer our original question; although MTL is required for conscious awareness of oldness, the reverse is not true: MTL can distinguish between old and new outside of consciousness.

We also investigated the following hypothesis: if a region in the brain registers the objective nature of the stimulus, then activity in this region should increase the discriminability between "true old" and "true new" items and, thus, be highly predictive of an individual's memory performance. We investigated this idea by calculating the correlation across participants between true oldness activity in this region and recognition accuracy (D-prime scores). Confirming our hypothesis, the results revealed a highly significant correlation $(r=0.74 ; p=0.0016)$ (Fig. 2). At the same time, the more restricted comparison misses $>$ FAs did not show a significant correlation $(p=0.26)$, indicating that $\mathrm{D}$-prime does not correlate with error-related activity in the posterior MTL.

\section{Follow-up analyses}

In a series of follow-up analyses, we addressed the question of why participants make errors when there is a true oldness signal in the posterior MTL. A possible answer to this question is that recognition outcome is not only determined by the true oldness signal in the posterior MTL, but also by activity in other brain regions. Although we focused initially on regions showing oldness-related activity, we also identified an anterior MTL region, which included the anterior portion of the hippocampus, that was sensitive to perceived novelty [(CRs and misses) $>$ (hits and FAs); $p<0.001$, cluster size, 10]. Similar to the posterior MTL region, an ANOVA of the mean anterior MTL activity revealed no significant true by perceived oldness interaction ( $p=$ $0.75)$. Furthermore, there also was no significant difference in mean activity between items perceived as new, CRs, and misses

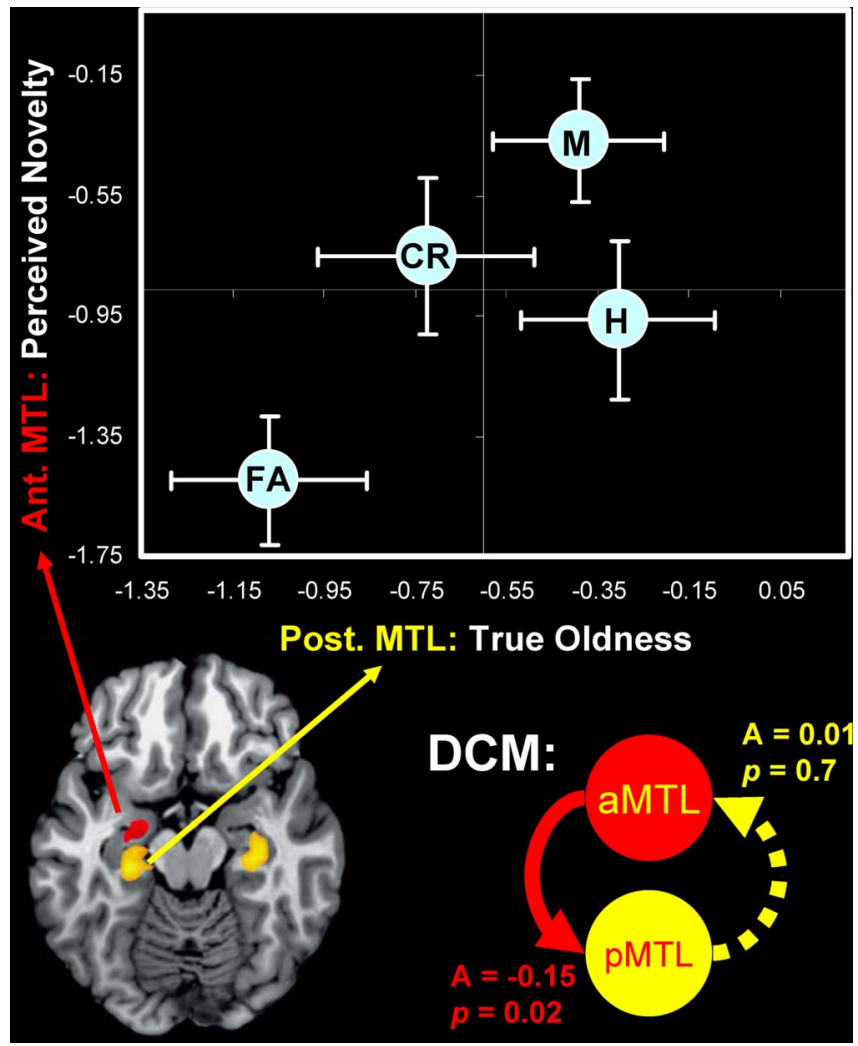

Figure 3. Top, Recognition performance is codetermined by true oldness activity in posterior MTL and perceived novelty activity in anterior MTL. The dots denote the different trial types [hit $(H)$, miss $(M), C R$, and $F A]$ as a function of activity in the two MTL regions. The horizontal and vertical bars indicate SEM. Bottom, The DCM analysis indicated a significant negative coupling from the left anterior MTL to the left posterior MTL, but not vice versa.

$(p=0.11)$. The locations of the true oldness and perceived novelty MTL regions are shown in Figure 3. Thus, a possible explanation of why recognition performance was not as good as predicted by true oldness activity in the posterior MTL is that performance was also influenced by perceived novelty activity in the anterior MTL.

To investigate this hypothesis, we conducted three analyses. First, we compared activity in the left anterior and posterior MTL regions for the four recognition outcomes. As indicated by the top part of Figure 3, true oldness activity was similar for hits and misses, whereas perceived novelty activity was reliably greater for misses $(p=0.032)$. These results suggest that, despite a high level of true oldness activity, missed items were misclassified because of a high level of perceived novelty activity. Critically, the $x$ - and $y$-axes included in Figure 3 illustrate that the four different trial types can be classified by simply combining the two MTL signals. Although the separation between CRs and hits may appear to be small along the $y$-axis (perceived novelty), this difference was in fact significant $(p=0.030)$. Likewise, the differences between misses and hits in the anterior MTL $(p=0.035)$, and misses and CRs in the posterior MTL $(p=0.046)$ were also significant.

Second, we investigated to what extent the probability of responding "old" or "new" in each individual trial was determined by the level of activity in the two MTL regions using a logistic regression analysis based on individual trial activity. The results indicated that activity in posterior MTL significantly increased the probability of classifying old items as "old" ( $p=0.0059)$, whereas activity in anterior MTL significantly increased the probability of classifying old items as "new" ( $p=0.0046)$. These 
results indicate that true oldness and perceived novelty signals made significant and independent contributions to recognition memory decisions.

Finally, we investigated the functional connectivity between the anterior and posterior MTL regions using the DCM integrated in SPM2. As shown in the bottom part of Figure 3, the results revealed a significant negative coupling from the left anterior MTL to the left posterior MTL $(A=-0.15$; SD, $0.22 ; p=$ $0.022)$, but not vice versa $(A=0.01 ; \mathrm{SD}, 0.11 ; p=0.72)$. This result suggests an opposing relationship between the true oldness signal in the posterior MTL and the perceived novelty signal in the anterior MTL. Together, the three analyses support our hypothesis that, despite having a true oldness signal in posterior MTL, participants make errors because their performance is also influenced by an opposing perceived novelty signal.

\section{Discussion}

\section{True oldness activity in posterior MTL}

By comparing MTL activity for all four recognition outcomes, we found a posterior MTL region that distinguishes between old and new items independently of consciousness. In other words, posterior MTL was more sensitive to the true oldness of the stimuli than to participants' awareness of it. This finding is striking because it shows that there is a brain region that can detect objective differences between old and new items that are not accessible to consciousness.

However, an alternative explanation to this finding is that posterior MTL activity for old stimuli that were incorrectly perceived as new (misses) simply reflects a weaker form of conscious memory; that is, activity for misses may have been just below the response threshold so that participants were unsure, leading them to make a "new" response. However, in that case, one would expect less activity in this region for incorrect (misses) compared with correct (hits) old responses. In fact, posterior MTL activity was very similar for hits and misses, and a direct comparison did not reveal any significant differences. Moreover, confirming its exquisite sensitivity to the objective nature of the stimulus, activity in posterior MTL was strongly correlated with individual memory performance (Fig. 2).

The present finding of true oldness activity in the posterior MTL seems to disagree with previous studies that associated activity in the posterior MTL with conscious memory processes (Schacter et al., 1996; Eldridge et al., 2000; Prince et al., 2005). At the same time, our findings are in line with evidence that posterior MTL regions can be immune to memory illusions (Cabeza et al., 2001). Although we do not have a clear-cut explanation for this discrepancy in findings, the activations reported in these different studies may involve functionally distinct areas of the posterior MTL (e.g., parahippocampal vs hippocampal). In general, our findings indicate that certain portions of the MTL are very sensitive to the veridical history of past events. This idea fits well with current models of MTL function (Eichenbaum, 2004; Squire et al., 2004) and with the notion that the MTL is a brain module that automatically reactivates memory traces with the presentation of a memory cue (Moscovitch, 1995).

\section{Why do we make errors?}

Although consistent with the critical role of the MTL in memory, the finding that this region shows true oldness activity raises an important question: why do participants make errors when their own brains hold the correct answer? A possible explanation is that participants' behavior reflects the combined effects of multiple brain regions, and that while the posterior MTL region in Figure
$1 B$ is tracking the true oldness of the stimuli, other brain regions respond to aspects of the stimuli that are not diagnostic of true oldness. In fact, we identified not only the posterior MTL region that is sensitive to true oldness but also an anterior MTL region that is sensitive to perceived novelty (Fig. 3). This finding is consistent with functional neuroimaging and intracranial recording evidence showing that the anterior MTL is sensitive to stimulus novelty (Grunwald et al., 1998; Henson et al., 2003; Gonsalves et al., 2005). Thus, a possible explanation of why recognition performance was not as good as predicted by true oldness activity in posterior MTL is that performance was also influenced by perceived novelty activity in the anterior MTL.

To investigate this hypothesis, we conducted three analyses. In the first analysis, we compared activity in the left anterior and posterior MTL regions for the four recognition outcomes. The results were consistent with our hypothesis. Whereas true oldness activity was similar for hits and misses, perceived novelty activity was reliably greater for misses (Fig. 3, top). Thus, the results indicate that, despite eliciting high true oldness activity, some old items may have been misclassified as new, because they also elicited high perceived novelty activity.

Yet, it should be noted that the true oldness and perceived novelty patterns were not as clear-cut when considered separately. For instance, despite the fact that FAs were labeled as "old" by participants, these items showed much lower true oldness activity than all the other trial types. However, this inconsistency can be reconciled by the fact that FAs also showed extremely low perceived novelty activity. Thus, one may speculate that in the case of FAs, the depth of the perceived novelty signal outweighed the depth of the true oldness signal, biasing participants to make an old response. Critically, the $x$ - and $y$-axes included in Figure 3 illustrate that the four different trial types can be classified by simply combining the two MTL signals. As can be seen, the resulting quadrants in Figure 3 perfectly match the matrix with the four recognition outcomes depicted in Figure $1 \mathrm{~A}$. To account for more graded differences in oldness and recognition confidence, it would be necessary to consider more brain areas in addition to the anterior and posterior MTL regions identified in the present study.

The finding of a novelty signal in addition to an oldness signal in the MTL raises the question of whether novelty truly involves a different signal than oldness or whether it is just the reverse of the oldness signal. In other words, do the anterior and posterior MTL regions make separate contributions to recognition performance? The answer to this question was found in the second follow-up analysis. Here, we investigated to what extent the probability of responding "old" or "new" in each individual trial was determined by the level of activity in the two MTL regions. To this end, we conducted a logistic regression analysis based on individual trial activity. This analysis indicated that whereas activity in the posterior MTL region (true oldness) significantly increased the probability of classifying old items as "old," activity in the anterior MTL region (perceived novelty) significantly enhanced the probability of classifying old items as "new." Thus, these results indicate that the two MTL regions had an opposing effect on participants' memory decisions. Moreover, the fact that both MTL regions significantly predicted behavior indicates that posterior and anterior MTL make independent contributions to recognition memory. Yet, it is worth noting that a similar analysis for new items indicated that only anterior MTL activity ( $p=$ 0.0078 ), and not posterior MTL ( $p=0.73)$, significantly increased the probability of classifying new items as "new." More research is required to elucidate the contributions of the anterior 
MTL and its interactions with other brain regions, such as the frontal lobes, to the classification of new items.

Finally, in the third analysis we investigated the functional connectivity between the true oldness and perceived novelty MTL regions using the DCM integrated in SPM2. The results revealed a negative coupling from the left anterior MTL to the left posterior MTL, but not vice versa. These findings indicate a negative feedback mechanism in which true oldness activity in the posterior MTL is suppressed by perceived novelty activity in anterior MTL (Fig. 3, bottom). One explanation for this finding may reside in the link that exists between perceived novelty and episodic encoding. According to the novelty-encoding account, the level of perceived novelty of information determines whether that information will be encoded into longterm memory (Tulving et al., 1996). At the same time, studies have associated activity specifically in the anterior MTL (perceived novelty) with successful memory encoding (Daselaar et al., 2004; Prince et al., 2005). Hence, the finding that the anterior MTL inhibits activity in the posterior MTL may reflect a natural opposition between the episodic-encoding (anterior MTL) and episodic-retrieval (posterior MTL) networks.

The finding of a negative coupling between the two MTL regions converges with the logistic regression analyses indicating opposing relationships with behavior. Together, the three analyses indicated that the two MTL regions had opposing effects on memory performance, that their activity was negatively coupled, and that recognition performance was determined by the joint activity of these regions.

\section{Conclusion}

In summary, we found that MTL can detect objective differences between old and new items that are not accessible to consciousness. Confirming its sensitivity to the objective nature of the stimulus, true oldness activity in this region was strongly correlated with individual memory performance. At the same time, follow-up analyses indicated that memory errors were associated with activity in an anterior MTL region that signaled whether an item was consciously experienced as new (perceived novelty). Furthermore, the two MTL regions showed opposing relationships with behavior, and memory performance was determined by their joint activity. These findings show that participants' behavior reflects the combined effects of multiple brain regions.

More generally, our results indicate that portions of the MTL are extremely sensitive to the veridical history of past events. This idea fits well with current models of MTL function (Eichenbaum, 2004; Squire et al., 2004) and with the notion that MTL is a brain module that automatically reactivates memory traces with the presentation of a memory cue (Moscovitch, 1995). At the same time, similar to some lesion (Chun and Phelps, 1999) and neu- roimaging data (Henke et al., 2003; Degonda et al., 2005), the present finding challenges the idea that MTL memory functions are necessarily dependent on conscious awareness.

\section{References}

Buckner RL, Wheeler ME (2001) The cognitive neuroscience of remembering. Nat Rev Neurosci 2:624-634.

Cabeza R, Rao SM, Wagner AD, Mayer AR, Schacter DL (2001) Can medial temporal lobe regions distinguish true from false? An event-related fMRI study of veridical and illusory recognition memory. Proc Natl Acad Sci USA 98:4805-4810.

Chun MM, Phelps EA (1999) Memory deficits for implicit contextual information in amnesic subjects with hippocampal damage. Nat Neurosci 2:844-847.

Daselaar SM, Veltman DJ, Witter MP (2004) Common pathway in the medial temporal lobe for storage and recovery of words as revealed by eventrelated functional MRI. Hippocampus 14:163-169.

Degonda N, Mondadori CR, Bosshardt S, Schmidt CF, Boesiger P, Nitsch RM, Hock C, Henke K (2005) Implicit associative learning engages the hippocampus and interacts with explicit associative learning. Neuron 46:505-520.

Eichenbaum H (2004) Hippocampus: cognitive processes and neural representations that underlie declarative memory. Neuron 44:109-120.

Eldridge LL, Knowlton BJ, Furmanski CS, Bookheimer SY, Engel SA (2000) Remembering episodes: a selective role for the hippocampus during retrieval. Nat Neurosci 3:1149-1152.

Friston KJ, Harrison L, Penny W (2003) Dynamic causal modelling. NeuroImage 19:1273-1302.

Gonsalves BD, Kahn I, Curran T, Norman KA, Wagner AD (2005) Memory strength and repetition suppression: multimodal imaging of medial temporal cortical contributions to recognition. Neuron 47:751-761.

Grunwald T, Lehnertz K, Heinze HJ, Helmstaedter C, Elger CE (1998) Verbal novelty detection within the hippocampus proper. Proc Natl Acad Sci USA 95:3193-3197.

Henke K, Mondadori CR, Treyer V, Nitsch RM, Buck A, Hock C (2003) Nonconscious formation and reactivation of semantic associations by way of the medial temporal lobe. Neuropsychologia 41:863-876.

Henson RN, Cansino S, Herron JE, Robb WG, Rugg MD (2003) A familiarity signal in human anterior medial temporal cortex? Hippocampus 13:301-304.

Moscovitch M (1995) Recovered consciousness: a hypothesis concerning modularity and episodic memory. J Clin Exp Neuropsychol 17:276-290.

Prince SE, Daselaar SM, Cabeza R (2005) Neural correlates of relational memory: successful encoding and retrieval of semantic and perceptual associations. J Neurosci 25:1203-1210.

Rissman J, Gazzaley A, D’Esposito M (2004) Measuring functional connectivity during distinct stages of a cognitive task. NeuroImage 23:752-763.

Schacter DL, Alpert NM, Savage CR, Rauch SL, Albert MS (1996) Conscious recollection and the human hippocampal formation: evidence from positron emission tomography. Proc Natl Acad Sci USA 93:321-325.

Squire LR, Stark CE, Clark RE (2004) The medial temporal lobe. Annu Rev Neurosci 27:279-306.

Tulving E, Markowitsch HJ, Craik FM, Habib R, Houle S (1996) Novelty and familiarity activations in PET studies of memory encoding and retrieval. Cereb Cortex 6:71-79. 